\title{
States on Clifford Algebras
}

\author{
Erik Batslev* and André Verbeure** \\ Institut des Hautes Etudes Scientifiques \\ 91 Bures-sur-Yvette - France \\ Received July 10, 1967
}

\begin{abstract}
We study states on Clifford algebras from the point of view of $\mathrm{C}^{*}$. algebras. A criterium is given under which the odd-point functions vanish. A particular set of states, called quasi-free states is extensively studied and explicit representations are given; as an application we give an approximate calculation of the ground state of a Fermion system.
\end{abstract}

\section{Introduction}

Recently quantum field theory and statistical mechanies have been studied from the point of view of $\mathrm{C}^{*}$-algebras. The key idea is the following. The set of quasi-local observables of a physical system forms a $\mathrm{C}^{*}$-algebra, and the physical states of the system correspond to the states (positive linear functionals) on the $\mathrm{C}^{*}$-algebra.

The $\mathrm{C}^{*}$-algebra formed by the field variables of a Bose field is studied by D. Kastler [1] and D. W. Robinson [2].

In this paper we study states on the Clifford algebra formed by the field varables of a Fermion field.

In section II, containing the definition of a $\mathrm{C}^{*}$-Clifford algebra and several relevant notions originating from physical considerations we prove that the odd-point functions vanish for states invariant under a locally compact group, a property which was known for relativistic field theories.

In section III we give a definition of a particular class of states, called quasi-free states. This notion has been introduced by D. W. RoвINson [2] for Bose systems. Here we define such states for Fermion fields. It is proved that the set of quasi-free states can be described by means of the set of pairs $(R, S)$ of operators on the test function space (see theorem 2); gauge invariant quasi-free states are characterized by $S=0$ and translation invariant quasi-free states by convolution operators $R$ and $S$ defined by distributions whose Fourier transforms are essentially bounded.

* On leave from Matematisk Institut, University of Aarhus, Denmark.

** Aangesteld navorser van het Belgisch N. F. W. O. On leave from University of Louvain, Belgium. 
In section IV the translation invariant states are studied in detail, in particular an explicit construction is given for their representations in terms of Fock representation. In section $V$ the method is applied to states, described by invertible operators $R$ and $S$. In this way we extend the works of Araki and Wyss [3], and Shate and Stinespring [4] on representations of anticommutation relations.

Finally, we apply the theory of quasi-free states to a Fermion model. The ground states of this thermodynamical system are found by a variational procedure. An integral equation analogous to the one found for the Bardeen-Cooper-Schriefer model is obtained.

Representations of the anticommutation relations have been studied by other methods using infinite tensor products. We give the main reference to this approach in [6].

\section{Properties of Clifford Algebras}

Let $\mathfrak{M}$ be a prehilbert space with inner product $(.$, .), and let $\overline{\mathfrak{Z}}=\mathscr{H}$ be the completion of $\mathfrak{M}$. To every element $f \in \mathscr{H}$ there corresponds an element $f^{*}$ in the dual $\mathscr{H}^{*}$ of $\mathscr{H}$ defined by the mapping $g \in \mathscr{H} \rightarrow$ $\rightarrow(f, g)=f^{*}(g)$. Let $\mathfrak{M} *=\left\{f^{*} \in \mathscr{H} * \mid f \in \mathfrak{Z}\right\}$.

Definition 1. $\mathfrak{A}(\mathfrak{M})$, the Clifford algebra over $\mathfrak{M}$, is the algebra generated by the monomials $\left[h_{1}\right] \ldots\left[h_{n}\right], h_{i} \in \mathfrak{M} \cup \mathfrak{M}^{*}, 1 \leqq i \leqq n$, and the unit element 1, such that for $f, g \in \mathfrak{M}$ :

and

$$
[[f],[g]]_{+}=0,\left[[f],\left[g^{*}\right]\right]_{+}-(g, f) \mathbf{1}=\mathbf{0}
$$

$$
c_{1}[f]+c_{2}[g]-\left[c_{1} f+c_{2} g\right]=0 .
$$

The involution on $\mathfrak{A}(\mathfrak{M})$ is defined by $\mathbf{1}^{*}=\mathbf{1}$ and

and

$$
a=\sum_{\lambda} c_{\lambda}\left[h_{1 \lambda}\right] \ldots\left[h_{n(\lambda) \lambda}\right] \rightarrow a^{*}=\bar{c}_{\lambda}\left[h_{n(\lambda)}^{*}\right] \ldots\left[h_{1 \lambda}^{*}\right]
$$

$$
[h]^{* *}=h \quad h \in \mathscr{H} \cup \mathscr{H}^{*} .
$$

Since the Fock representation of $\mathfrak{A}(\mathfrak{M})$ (i.e. the representation by creation and annihilation operators on Fock space) is faithful (in fact all representations are faithful because the algebra $\mathfrak{A}(\mathfrak{R Z})$ is simple [4]) it induces a norm $\|\cdot\|$ on $\mathfrak{A}(\mathfrak{R})$, such that the closure of $\mathfrak{A}(\mathfrak{R})$ under this norm is a $\mathrm{C}^{*}$-algebra $\mathfrak{A}$. We call $\mathfrak{A}$ the $\mathrm{C}^{*}$-Clifford algebra over $\mathscr{H}$.

Definition 2. The monomials of even order generate a subalgebra $\mathfrak{A}$ even of $\mathfrak{A}$. Moreover let us denote by $\mathfrak{A}_{\text {odd }}$ the subspace generated by the monomials of odd order.

States of physical fermion systems are now represented by states on the $\mathrm{C}^{*}$-algebra $\mathfrak{A}$. In general a symmetry group is related to the algebra $\mathfrak{A}$, i.e. there is given a locally compact group $\mathfrak{G}$ and a continuous homomorphism $\tau$ of $\mathfrak{G}$ into the *automorphisms of $\mathfrak{A}$, mapping $g \in \mathfrak{G}$ into $\tau_{g}$ and 
we suppose that $\tau_{g}$ maps monomials into monomials of the same order. Let $\mathfrak{A}^{\prime}$ be the dual space of $\mathfrak{A}$ with the weak*-topology and let $\mathfrak{Q}^{++} \cong \mathfrak{U}^{\prime}$ be the (compact) set of states on $\mathfrak{A}$. Furthermore $\mathfrak{H}_{\mathfrak{S}}^{\prime+}=\left\{\varrho \in \mathfrak{A}^{\prime+} \mid \varrho\left(\tau_{g} \mathfrak{A}\right)\right.$ $=\varrho(A)$, for every $g \in \mathfrak{G}, A \in \mathfrak{A}\}$ is the set of $\mathfrak{G}$-invariant states on $\mathfrak{A}$.

According to the well known Gel'fand-Segal construction there corresponds to every state $\varrho$ a representation $\pi_{\ell}$ of $\mathfrak{A}$ on a Hilbert space $\mathfrak{S}_{\varrho}$ with cyclic vector $\Omega_{\varrho}$ such that

$$
\varrho(A)=\left(\Omega_{0}, \pi_{\varrho}(A) \Omega_{0}\right) \text { for } A \in \mathfrak{A} .
$$

If $\varrho \in \mathfrak{A}_{\mathfrak{S}}^{\prime+}$, there exists a unique unitary representation $U_{\varrho}$ of $\mathfrak{S}$ on $\mathfrak{G}_{\varrho}$, such that

$$
\begin{aligned}
& U_{\varrho}(g) \Omega_{Q}=\Omega_{\varrho} \quad \text { for all } g \in \mathfrak{G} \\
& U_{\varrho}(g) \pi_{\varrho}(A) U_{\varrho}\left(g^{-1}\right)=\pi_{\varrho}\left(\tau_{g}(A)\right) \text { for all } g \in \mathfrak{G}, A \in \mathfrak{A} .
\end{aligned}
$$

Finally, let $P_{Q}$ be the projection operator on the subspace of $\mathfrak{G}_{Q}$ invariant under the group $\mathfrak{G}$ (i.e. $U_{\varrho}(g) P_{Q} \mathfrak{S}_{Q}=P_{\varrho} \mathfrak{S}_{\varrho}$ for $g \in \mathfrak{G}$ ).

In the following definition we formulate the principle of locality for fermion systems with respect to the group $\mathfrak{F}$.

Definition 3. The algebra $\mathfrak{A}$ is called $\mathfrak{G}$-local if

$$
\begin{aligned}
& {\left[P_{\varrho} \pi_{\varrho}\left(A_{m}\right) P_{\varrho}, P_{\varrho} \pi_{\varrho}\left(B_{n}\right) P_{\varrho}\right]_{+}=0 \text { if } m \text { and } n \text { are odd }} \\
& {\left[P_{\varrho} \pi_{\varrho}\left(A_{m}\right) P_{\varrho}, P_{\varrho} \pi_{\varrho}\left(B_{n}\right) P_{\varrho}\right]_{-}=0 \text { if } m \text { and (or) } n \text { are even }}
\end{aligned}
$$

where $A_{m}$ and $B_{n}$ are monomials of order $m$ and $n$ respectively.

The following proposition may be used to give another formulation of locality with respect to a group $\mathfrak{G}$. The proof of this proposition can be given along the same lines as the proof of theorem 2.3 of reference [7].

Proposition 1. In order that $\left[P_{Q} \pi_{0}(A) P_{\varrho}, P_{0} \pi_{\varrho}(B) P_{\varrho}\right]_{ \pm}=0$ for $A$, $B \in \mathfrak{A}$ and $A=A^{*} ; B=B^{*}$ it is necessary and sufficient that for every $\varrho \in \mathfrak{A}_{\mathfrak{S}}^{\prime+} ; \inf _{A^{\prime}}\left|\varrho\left(\left[A^{\prime}, B\right]_{ \pm}\right)\right|=0$ where $A^{\prime}$ runs over the convex hull of $\left\{\tau_{g} A \mid g \in A\right\}$.

For relativistic Fermion fields it is well known [8] that odd-point Wightman functions vanish. Here we give a more general but elementary proof of this property. Another proof can be found in reference [9]. First we need

Definition 4. The algebra $\mathfrak{A}$ is called $\mathfrak{G}$-abelian if for all $\varrho \in \mathfrak{X}_{\mathfrak{G}}^{\prime+}$ the von Neumann algebra generated by $P_{\varrho} \pi_{\varrho}(\mathfrak{H}) P_{\varrho}$ is abelian.

Theorem 1. If $\mathfrak{A}$ is $\mathfrak{G}$-local and $\varrho \in \mathfrak{U}_{\mathfrak{G}}^{\prime+}$ then $\mathfrak{A}$ is $\mathfrak{G}$-abelian and in particular $\varrho\left(\mathfrak{A}_{\text {odd }}\right)=0$.

Proof $^{1}$. From definition 3, it follows that it suffices to prove that $P_{\varrho} \pi_{\varrho}\left(\mathfrak{A}_{\text {odd }}\right) P_{\varrho}$ is abelian. For $m=0,1,2 \ldots$ we have

where

$$
M M^{*}+M^{*} M=0
$$

$$
M=P_{\varrho} \pi_{\varrho}\left(A_{2 m+1}\right) P_{\varrho}
$$

1 We are indebted to D. W. Robinson for the first idea of this proof. 
$M M^{*}$ and $M^{*} M$ are positive operators, hence

$$
M M^{*}=M^{*} M=0 \text {. }
$$

This implies $M=P_{\varrho} \pi_{\varrho}\left(A_{2 m+1}\right) P_{\varrho}=0$, therefore $P_{\varrho} \pi_{\varrho}\left(\mathfrak{A}_{\text {odd }}\right) P_{\varrho}$ is abelian and also

$$
\left(\Omega_{\varrho}, P_{\varrho} \pi_{\varrho}\left(A_{2 m+1}\right) P_{\varrho} \Omega_{\varrho}\right)=\varrho\left(A_{2 m+1}\right)=0 \quad \text { q.e.d. }
$$

\section{Quasi-free States}

Let $\mathscr{H}$ be a separable Hilbert space with elements $f, g, \ldots$, inner product $(.,$.$) and conjugation - . We consider bounded linear operators$ on $\mathscr{H}$ denoted by $A, B, \ldots$ There is a one-to-one correspondence between operators $A$ and bounded sesqui-linear forms $(f, A g)$.

Definition 5. The transposed operator $A^{\prime}$ of an operator $A$ is defined by its sesquilinear form

$$
\left(f, A^{\prime} g\right)=(\bar{g}, A \bar{f})
$$

the complex conjugate operator $\bar{A}$ is defined by

$$
(f, \bar{A} g)=(f, \overline{A \bar{g}})
$$

the adjoint operator $A^{*}$ is given by

$$
\left(f, A^{*} g\right)=(A f, g) \text {. }
$$

For an integral operator $K$ with kernel $K(x, y)$, the operators $K^{\prime}, \bar{K}$ and $K^{*}$ correspond to the kernels $K(y, x), \overline{K(x, y)}$, and $\overline{K(y, x)}$ respectively.

One verifies the relations

$$
\begin{aligned}
A^{*}=\bar{A}^{\prime}=\bar{A}^{\prime} ; A^{\prime}= & \bar{A}^{*}=\bar{A}^{*} ; \bar{A}=A^{* \prime}=A^{\prime *} ; \bar{A}=A^{\prime \prime}=A^{* *}=A ; \\
& (A B)^{\prime}=B^{\prime} A^{\prime} ; \overline{A B}=\bar{A} \bar{B} .
\end{aligned}
$$

We use the notation $\eta_{A}=\{f \in \mathscr{H} \mid A f=0\} \mathfrak{R}_{A}=\{g=A f / f \in \mathscr{H}\}$. The operator $A^{-1}$ is defined by $\mathfrak{D}_{A^{-1}}=\Re_{A}$ and $A^{-1} A g=g$ for $g \in \eta \frac{1}{A} . A$ is said to be invertible, if $A^{-1}$ is a bounded operator on $\mathscr{H}$.

Now we define a particular class of states which we call quasi-free states. We need first

Definition 6. Let $\varrho$ be a state on $\mathfrak{A}$ such that $\varrho\left(\mathfrak{A}_{\text {odd }}\right)=0$, so that $\varrho$ is determined by its values on the even monomials. The truncated functions or correlation functions $\varrho^{T}$ of $\varrho$ are defined by the following recursive formula

$$
\varrho\left(Q_{1} \ldots Q_{n}\right)=\sum(-1)^{\eta_{i}} \varrho^{T}\left(Q_{i_{1}} \ldots\right) \ldots \varrho^{T}\left(\ldots Q_{i_{n}}\right)
$$

where $Q_{i}=\left[h_{i}\right], h_{i} \in \mathscr{H} \cup \mathscr{H}^{*}$, the sum is over all possible partitions of the $Q_{i}$, the first factors in the brackets as well as the factors in each bracket occur in natural order, and $\eta_{i}$ is the sign of the permutation

$$
\left(\begin{array}{l}
1 \ldots n \\
i_{1} \ldots i_{n}
\end{array}\right) \text {. }
$$


The state $\varrho$ is completely determined by its truncated functions $\varrho^{T}$. Now we can give

Definition $\%$. A quasi-free state $\varrho$ on $\mathfrak{A}$ is a state which satisfies the conditions :

$$
\varrho\left(\mathfrak{A}_{\text {odd }}\right)=0 ; \varrho^{T}\left(Q_{1} \ldots Q_{2 n}\right)=0 \text { for } n>1 .
$$

Hence the quasi-free state $\varrho$ is completely determined by the following pair of bounded sesqui-linear functionals of $f$ and $g$ or by the corresponding operators $R$ and $S$ on $\mathscr{H}$.

$$
\begin{aligned}
\varrho\left(\left[f^{*}\right][g]\right) & =\varrho^{T}\left(\left[f^{*}\right][g]\right)=(f, R g) \\
\varrho([\bar{f}][g]) & =\varrho^{T}([\bar{f}][g])=(f, S g) .
\end{aligned}
$$

Now we give conditions on $R$ and $S$ in order that (1) and (2) define a quasi-free state.

First we denote by $Q-\mathfrak{F}$ the set of quasi-free states and by $\mathfrak{O}$ the set of pairs of operators $(R, S)$, which define a state $\varrho \in Q-\mathfrak{F}$ by $(1)$ and (2).

In order to obtain a manageable subset of $Q-\mathfrak{F}$ we impose the condition that either

or

$\left(\mathrm{i}^{\prime}\right) \mathfrak{S}_{R^{-1} S^{*}}$ is dense in $\mathscr{H}$

We define

$\left(\mathrm{i}^{\prime \prime}\right) \mathfrak{S}_{\left(1-R^{\prime}\right)^{-1} S}$ is dense in $\mathscr{H}$.

$$
\begin{gathered}
\mathcal{O}_{1}^{\prime}=\left\{(R, S) \in \mathcal{O} \mid\left(\mathrm{i}^{\prime}\right) \text { holds }\right\}, \mathfrak{O}_{1}^{\prime \prime}=\left\{(R, S) \in \mathcal{O} \mid\left(\mathrm{i}^{\prime \prime}\right) \text { holds }\right\}, \\
\mathfrak{O}_{1}=\mathfrak{O}_{1}^{\prime} \cup \mathcal{O}_{1}^{\prime \prime}, Q-\mathfrak{F}_{1}=\left\{\varrho \in Q-\mathfrak{F} \mid(R, S) \in \mathcal{O}_{1}\right\} .
\end{gathered}
$$

Lemma 1. One has $(R, S) \in \mathcal{O}_{1}^{\prime}$ if $\mathfrak{R}_{R}$ is closed $\left(\mathfrak{S}_{R^{-1} S^{*}}=\mathscr{H}\right)$ or $\mathfrak{R}_{S^{*}}$ is closed or $[R, S]=\left[S, S^{*}\right]=0$. Similarly, $(R, S) \in \mathcal{O}_{1}^{\prime \prime}$ if $\mathfrak{R}_{1-R^{\prime}}$, or $\mathfrak{R}_{S}$ is closed, or $[R, S]=\left[S, S^{*}\right]=0$.

Proof. It follows from (1) and (2) by Schwarz's inequality, that

$$
\varrho\left(\left[f^{*}\right][g]\right) \leqq \varrho\left(\left[f^{*}\right][f]\right)^{1 / 2} \varrho\left([g]\left[g^{*}\right]\right)^{1 / 2}
$$

and consequently $\eta_{R} \subseteq \eta_{S}$ and $\eta_{1-R^{\prime}} \leqq \eta_{S^{*}}$. The rest follows by a simple argument, using that $\overline{\mathfrak{R}}_{R^{*}}=\eta_{\bar{S}}$, and the fact that $\mathfrak{R}_{S^{*}}$ is closed if $\mathfrak{R}_{S}$ is closed.

We characterize the set $\mathfrak{O}_{1}$ in the following theorem.

Theorem 2. The pair of operators $(R, S)$ belongs to $\mathfrak{O}_{1}^{\prime}\left(\mathcal{O}_{1}^{\prime \prime}\right)$ if and only if it satisfies $\mathrm{i}^{\prime}, \mathrm{ii}^{\prime}$, iii and iv $\left(\mathrm{i}^{\prime \prime}\right.$, $\mathrm{ii}^{\prime \prime}$, iii and $\left.\mathrm{iv}\right)$ where $\left(\mathrm{i}^{\prime}\right)$ and $\left(\mathrm{i}^{\prime \prime}\right)$ are as above and one has

(ii') $X=1-R^{\prime}-S R^{-1} S^{*} \geqq 0$ on $\mathfrak{P}_{R^{-1} S^{*}}$

(ii') $Y=R-S^{*}\left(1-R^{\prime}\right)^{-1} S \geqq 0$ on $\mathfrak{P}_{\left(1-R^{\prime}\right)^{-1} S}$

(iii) $R=R^{*} ; 0 \leqq R \leqq 1$

(iv) $S^{\prime}=-S$. 
If either $\mathfrak{R}_{R}$ and $\mathfrak{R}_{1-R^{\prime}}$ are closed, or $\mathfrak{R}_{S}$ is closed, or $[R, S]=0$, $\left[S, S^{*}\right]=0$, then $\left(\mathrm{ii}^{\prime}\right)$ and $\left(\mathrm{ii}^{\prime \prime}\right)$ are equivalent.

Proof. Suppose that $(R, S) \in \mathfrak{O}_{1}$.

Condition (iv) follows from (2) and the anticommutation relations.

According to a theorem of RoBINson [2] the condition $\varrho(A) \geqq 0$ for $A \in \mathfrak{A}$, is equivalent to

$$
\varrho\left(\left(\left[f^{*}\right]+[\bar{g}]\right)\left([f]+\left[\bar{g}^{*}\right]\right)\right) \geqq 0 \text { for } f, g \in \mathscr{H} .
$$

By (1)-(2) and the anticommutation relations (3) becomes

$$
(f, R f)+\left(f, S^{*} g\right)+(g, S f)+\left(g,\left(1-R^{\prime}\right) g\right) \geqq 0 \text { for } f, g .
$$

For $g=0$ we obtain $R \geqq 0$; for $f=0 R \leqq 1$, hence (iii). The inequality (4) is equivalent to

$$
\left(\begin{array}{l}
f \\
g
\end{array}\right)\left(\begin{array}{ll}
R & S^{*} \\
S & 1-R^{\prime}
\end{array}\right)\left(\begin{array}{l}
f \\
g
\end{array}\right) \geqq 0 \quad \text { for } f \oplus g \text { on } \mathscr{H} \oplus \mathscr{H} .
$$

For $f \in \mathscr{H}, g \in \mathfrak{S}_{R^{-1} S^{*}}$ we have

$$
\left(\begin{array}{l}
f \\
g
\end{array}\right)\left(\begin{array}{ll}
R & S^{*} \\
S & 1-R^{\prime}
\end{array}\right)\left(\begin{array}{l}
f \\
g
\end{array}\right)=\left(\begin{array}{l}
f \\
g
\end{array}\right)\left(\begin{array}{ll}
R & S^{*} \\
S & S R^{-1} S^{*}
\end{array}\right)\left(\begin{array}{l}
f \\
g
\end{array}\right)+\left(\begin{array}{l}
f \\
g
\end{array}\right)\left(\begin{array}{ll}
0 & 0 \\
0 & X
\end{array}\right)\left(\begin{array}{l}
f \\
g
\end{array}\right) .
$$

Set $g_{1}=R^{-1} S^{*} g$, then $(6)$ becomes

$$
\left(\begin{array}{l}
f \\
g
\end{array}\right)\left(\begin{array}{ll}
R & S^{*} \\
S & 1-R^{\prime}
\end{array}\right)\left(\begin{array}{l}
f \\
g
\end{array}\right)=\left(f+g_{1}, R\left(f+g_{1}\right)\right)+(g, X g)
$$

From $\left(6^{\prime}\right)$ follows

$$
\left(\begin{array}{l}
f \\
g
\end{array}\right)\left(\begin{array}{ll}
R & S^{*} \\
S & S R^{-1} S^{*}
\end{array}\right)\left(\begin{array}{l}
f \\
g
\end{array}\right) \geqq 0 \quad \text { for } f \in \mathscr{H}, g \in \mathfrak{S}_{R^{-1} S^{*}}
$$

and the form is equal to zero for $f=-R^{-1} S^{*} g$.

For $f \in \mathfrak{S}_{\left(1-R^{\prime}\right)^{-1} S}, g \in \mathscr{H}$ we have

$$
\left(\begin{array}{l}
f \\
g
\end{array}\right)\left(\begin{array}{ll}
R & S^{*} \\
S & 1-R^{\prime}
\end{array}\right)\left(\begin{array}{l}
f \\
g
\end{array}\right)=\left(\begin{array}{l}
f \\
g
\end{array}\right)\left(\begin{array}{cr}
S^{*}\left(1-R^{\prime}\right)^{-1} S & S^{*} \\
S & 1-R^{\prime}
\end{array}\right)\left(\begin{array}{l}
f \\
g
\end{array}\right)+\left(\begin{array}{l}
f \\
g
\end{array}\right)\left(\begin{array}{ll}
Y & 0 \\
0 & 0
\end{array}\right)\left(\begin{array}{l}
f \\
g
\end{array}\right) .
$$

Let $f_{1}=\left(1-R^{\prime}\right)^{-1} S f$ then (8) becomes

$$
\left(\begin{array}{l}
f \\
g
\end{array}\right)\left(\begin{array}{ll}
R & S^{*} \\
S & 1-R^{\prime}
\end{array}\right)\left(\begin{array}{l}
f \\
g
\end{array}\right)=\left(f_{1}+g,\left(1-R^{\prime}\right)\left(f_{1}+g\right)\right)+(f, Y f) .
$$

From $\left(8^{\prime}\right)$ follows

$$
\left(\begin{array}{l}
f \\
g
\end{array}\right)\left(\begin{array}{cr}
S^{*}\left(1-R^{\prime}\right) S & S^{*} \\
S & 1-R^{\prime}
\end{array}\right)\left(\begin{array}{l}
f \\
g
\end{array}\right) \geqq 0 \text { for } f \in \mathfrak{S}_{\left(1-R^{\prime}\right)^{-1} S}, g \in \mathscr{H},
$$

and this form is equal to zero for $g=-\left(1-R^{\prime}\right)^{-1} S f$.

It follows from $(6)-\left(6^{\prime}\right)$ and $(7)$ that

$$
(g, X g) \geqq 0 \quad \text { for } \mathfrak{P}_{R^{-1} S^{*}}, \text { hence }\left(\mathrm{ii}^{\prime}\right)
$$

and from $(8)-\left(8^{\prime}\right)$ and $(9)$ that

$$
(f, Y f) \geqq 0 \text { for } f \in \mathfrak{S}_{\left(1-R^{\prime}\right)^{-1} S} \text {, hence }\left(\mathrm{ii}^{\prime \prime}\right) \text {. }
$$

Reversing the arguments it is easily checked that the conditions (i'), (ii'), (iii), (iv) or $\left(\mathrm{i}^{\prime \prime}\right),\left(\mathrm{ii}^{\prime \prime}\right)$, (iii), (iv) determine a quasi-free state $\varrho \in Q-\mathfrak{F}_{1}$. 
In the following two lemmas we characterize states invariant under translations.

Lemma 2. In order that the state $\varrho \in Q-\mathfrak{F}$ be invariant under the transformations

$$
\begin{aligned}
{[h] } & \rightarrow e^{i \alpha}[h] \quad h \in \mathscr{H} ; \alpha \in \mathbb{C} \\
{\left[h^{*}\right] } & \rightarrow e^{-i \alpha}\left[h^{*}\right]
\end{aligned}
$$

it is necessary and sufficient that $S=0$.

Proof. The proof of this Lemma is immediate.

Lemma 3. In order that the state $\varrho \in Q-\mathfrak{F}$ is invariant under the group $R^{v}$ of translations it is necessary and sufficient that the operators $R$ and $S$ on $\mathscr{H}=L^{2}\left(R^{v}\right)$ are convolution operators by distributions whose Fourier transforms are $L^{\infty}\left(R^{v}\right)$ functions.

Proof. Suppose that the Clifford algebra $\mathfrak{A}$ is built on $\mathscr{H}=L^{2}\left(R^{v}\right)$. By (1), (2) $R$ and $S$ are operators on $L^{2}\left(R^{\nu}\right)$. The symmetry group of the system is $\mathfrak{G}=R^{v}$ acting on $\mathfrak{A}$ by the automorphisms $\tau_{x}$ defined by

$$
\tau_{x}[h]=\left[h_{x}\right], h \in \mathscr{H}, x \in R^{v}
$$

where $h_{x}$ is the translated function of $h$, i.e. $h_{x}(y)=h(y-x)$.

The quasi-free state $\varrho$ is invariant under $R^{\nu}$ if and only if for every $x \in R^{v}$

and

$$
\left(f_{x}, R g_{x}\right)=(f, R g)
$$

$$
\left(f_{x}, S g_{x}\right)=(f, S g) \text {. }
$$

The translated operator $A_{x}$ of $A$ is defined by

$$
A_{x} g_{x}=A g \text { for all } x \in R^{v} \text { and } g \in L^{2}\left(R^{\nu}\right)
$$

and $A$ is called translation invariant if and only if

$$
A_{x}=A \text { for all } x \in R^{v} \text {. }
$$

It is easy to verify that (13) and (14) are equivalent with $R_{x}=R$ and $S_{x}=S$ or in other words that $R$ and $S$ are translation invariant operators. Such operators are characterized by Hörmander (cf. [10]) as follows. A bounded operator on $L^{2}\left(R^{v}\right)$ is translation invariant if and only if it is a convolution operator by a distribution whose Fourier transform belongs to $L^{\infty}\left(R^{v}\right)$, i.e. for $R$ and $S$

$$
R(f)=r * f ; S(f)=s * f \text { for } f \in L^{2}\left(R^{\nu}\right),
$$

where the Fourier transforms $\tilde{r}$ and $\tilde{s}$ of $r$ and $s$ are $L^{\infty}\left(R^{v}\right)$ functions. In momentum space

where

$$
\widetilde{R}(\tilde{f})=\tilde{r} \cdot \tilde{f} ; \tilde{S}(\tilde{f})=\tilde{s} \cdot \tilde{f} \text { for } \tilde{f} \in L^{2}\left(R^{v}\right)
$$

$$
\widetilde{R}(\tilde{f})=\overparen{R(f)} \text { and } \tilde{S}(\tilde{f})=\overparen{S(f)}
$$


Corollary. If $\varrho$ is a translation invariant quasi-free state, then the corresponding pair of operators $R$ and $S$ commutes, and moreover $S$ is a normal operator.

The proof is an immediate consequence of Lemma 3.

We are interested in representations $\phi$ of the $\mathrm{C}^{*}$-Clifford algebra $\mathfrak{A}$ induced by quasi-free states. The Gel'fand-Segal construction yields an abstract characterization. Our aim is to obtain a more explicit representation in terms of creation and annihilation operators on Fock space. We treat the cases $X \equiv 0$ (or $Y \equiv 0$ ) and $X \neq 0$ (or $Y \neq 0$ ) separately. It turns out, that these cases correspond to irreducible and reducible representations or to pure states and mixtures, respectively.

We denote by $\varphi(f)$ and $\varphi^{*}(f)$ the annihilation and creation operators of the Fock representation $\pi_{F}$ on Fock space $H_{F}$ with cyclic element (vacuum state) $\Omega_{F}$ defined by

$$
\begin{gathered}
\varphi(f) \Omega_{F}=0 \text { for all } f \in \mathscr{H} ; \\
\varphi(\bar{A} f)=\pi_{F}\left(\left[(A f)^{*}\right]\right) ; \varphi^{*}(B f)=\pi_{F}([B f]) .
\end{gathered}
$$

We shall use the following notation; where $\Phi$ is the representation associated with the quasi-free state $\varrho$.

$$
\begin{array}{rll}
\phi_{0}(f)=\phi([h]) & \text { if } & h=f \in \mathscr{H} \\
\phi_{0}^{+}(f)=\phi([h]) & \text { if } & h^{*}=f \in \mathscr{H} .
\end{array}
$$

It is clear, that $\phi_{0}$ and $\phi_{0}^{+}$determine completely the representation $\phi$. In what follows, we consider the case $X \geqq 0$; the case $Y \geqq 0$ is analogous.

\section{Translation Invariant States}

In section II we proved that if $\varrho$ is a quasi-free invariant state, the corresponding operators $R$ and $S$ commute and $S$ is a normal operator. In this section we want to study the representations of states for which $R$ and $S$ have these properties. We call them in general commutative. This class of states contains at least the translation invariant states.

Let $S=W|S|$ be the polar decomposition of $S$. Because $S$ is normal, it follows [11] that $W$ may be taken unitary and such that $W$ and $S$ commute with each other and with all operators that commute with $S$ and $S *$. Therefore

$$
\begin{aligned}
W|S| & =|S| W \\
R W & =W R \\
|S| R & =R|S| .
\end{aligned}
$$

From (iv) and (15) it follows that

$$
|S|^{\prime}=|S| \text { and }^{2} W \text { may be chosen satisfying } W^{\prime}=-W \text {. }
$$

We remark that for the class of states for which $[R, S]=\left[S, S^{*}\right]=0$, the set $\mathfrak{S}_{R^{-1} S^{*}}$ is always dense.

\footnotetext{
${ }^{2}$ Notice that this is possible only if $\operatorname{dim} \eta_{S}$ is even or infinite.
} 


\section{A. The case $X=0$}

First we analyze the relevant pairs of operators $(R, S)$ which satisfy (iii), (iv) and $X=0$ in the commutative case.

From $X=0$ on $\mathfrak{S}_{R^{-1} S^{*}}$, it follows that $X$ can be extended to a bounded operator on $\mathscr{H}$; using the commutativity of $R$ and $S$ we have

$$
R\left(1-R^{\prime}\right)=\left(1-R^{\prime}\right) R=S S^{*}
$$

or

$$
\left[R, R^{\prime}\right]=0 \text {. }
$$

Also $X^{\prime}=0$, and this yields

$$
R=R^{\prime}
$$

and immediately

$$
|S|=[R(1-R)]^{1 / 2} .
$$

We summarize the result in

Lemma 4. The solution of (iii), (iv) and $X=0$ in the commutative case is the set of pairs $(R, S=W|S|)$ such that

$$
\begin{aligned}
|S| & =[R(1-R)]^{1 / 2} \\
R & =R^{*} ; 0 \leqq R \leqq 1 \\
W W^{*} & =W^{*} W=1 \\
W^{\prime} & =-W \\
R^{\prime} & =R\left(|S|^{\prime}=|S|\right) \\
W R & =R W .
\end{aligned}
$$

In the next Lemma, we propose an explicit representation of the quasi-free state $\varrho$ on $H_{F}$ via certain operators $A$ and $B$ on $\mathscr{H}$. Afterwards we show that such a representation is actually possible by finding $A$ and $B$ in terms of $R$ and $S$. Now, we look for a representation of the quasi-free state $\varrho$ on $H_{F}$ by making the following Ansatz.

Let $A$ and $B$ be operators on $\mathscr{H}$ and set for $f \in \mathscr{H}$

$$
\begin{aligned}
\phi_{0}(f) & =\varphi(A f)+\varphi^{*}(B f) \\
\phi_{0}^{*}(f) & =\varphi(\bar{B} f)+\varphi^{*}(\bar{A} f) .
\end{aligned}
$$

It is easy to verify, that (21) and (22) generate a representation $\phi$ of $\mathfrak{A}$ if $A$ and $B$ satisfy

$$
\begin{aligned}
& A^{*} A+B^{*} B=1 \\
& A^{\prime} B+B^{\prime} A=0 .
\end{aligned}
$$

A simple calculation shows that if $A$ and $B$ satisfy moreover

$$
\begin{aligned}
& R=B^{*} B \\
& S=A^{\prime} B
\end{aligned}
$$

then $\varrho$ coincides on $\mathfrak{A}_{2}$ with the vector state associated with the cyclic element $\Omega_{F}$ and the representation $\phi$, i.e.

$$
\varrho(A)=\left(\Omega_{F}, \phi(A) \Omega_{F}\right), A \in \mathfrak{A}_{2}
$$

where $\mathfrak{A}_{\mathbf{2}}$ in generated by $\mathbf{1}$ and the monomials of order two. 
It follows from the properties of the Fock representation, that this vector state is quasi-free, and hence the identity (27) holds for all $A \in \mathfrak{R}$.

We summarize this discussion in

Lemma 5. Let $\varrho$ be the quasi-free state defined by the pair of operators $(R, S)$ with $X=0$, and let $(A, B)$ be a solution of (23)-(26). Then $\varrho$ is the vector state defined by (27) associated with the cyclic element $\Omega_{F}$ and the representation $\phi$ defined by (21) and (22).

We notice without going into details that certain additional conditions on $A$ and $B$ are required in order that $[R, S]=\left[S, S^{*}\right]=0$, and that $\mathfrak{S}_{R^{-1} S^{*}}$ is dense if and only if $\mathfrak{S}_{A^{-1}} \bar{B}$ is dense.

From Lemma 5 we see that if $X=0$ and $(R, S) \in \mathcal{O}^{\prime}$, it suffices in order to represent $\varrho$ on Fock space to solve the equetions (23)-(26) for $A$ and $B$. The solution is given in

Lemma 6. Suppose that $(R, S)$ is a pair of operators satisfying (iii), (iv) and $X=0$. Then a solution of (23)-(26) is given by

$$
\begin{aligned}
& A=-V^{*} W \sqrt{1-R} \\
& B=V \sqrt{R}
\end{aligned}
$$

where $V$ is an arbitrary unitary operator.

Proof. First we notice that (24) follows from (26) and (iv).

Let us take first $B=B^{*}$ then (25) gives

$$
B=\sqrt{R} \text {. }
$$

Then (15) - (20) and (iv) yield for (26)

$$
\sqrt{R} A=-W \sqrt{R(1-R)}
$$

and (16) assures us that $A=-W \sqrt{1-R}$ is a particular solution of (26). Multiplying $B=\sqrt{R}$ by an arbitrary unitary operator $V$ we obtain a class of solutions given by (28) and (29).

As a consequence of Lemmas 4-6 we obtain the following result. We omit the proof of the last statement, which follows from the analogous property of the Fock representation.

Theorem 3. Let $\varrho$ be a quasi-free state on $\mathfrak{A}$ corresponding to the operators $(R, S)$ defined in Theorem 2, such that $X=0,\left[S, S^{*}\right]=0$, and $[R, S]=0$. Then $\varrho$ corresponds to a representation $\phi$ of $\mathfrak{A}$ on Fock space $H_{F}$ where $\phi$ is defined by

$$
\begin{array}{lll}
\phi([h])=\varphi(-W \sqrt{1-R} f)+\varphi^{*}(\sqrt{R} f) & \text { if } & h=f \in \mathscr{H} \\
\phi([h])=\varphi(\sqrt{R} f)+\varphi^{*}(-\bar{W} \sqrt{1-R} f) & \text { if } & h^{*}=f \in \mathscr{H}
\end{array}
$$

i.e. $\varrho(A)=\left(\Omega_{F}, \phi(A) \Omega_{F}\right)$ for all $A \in \mathfrak{Q}$.

The representation $\phi$ is irreducible. 


\section{B. The case $X \geqq 0, X \neq 0$}

In analogy with the case $X=0$, we start writing down a possible candidate for a representation of $\mathfrak{A}$ associated with the quasi-free state $\varrho$. Afterwards we prove that it can be realized.

Let us take two copies of Fock representation with creation and annihilation operators $\varphi_{i}, \varphi_{i}^{*}(i=1,2)$, acting on Fock space $H_{F_{i}}(i=1,2)$ with vacuum elements $\Omega_{F_{i}}(i=1,2)$.

Let the quasi-free state $\varrho$ be defined by the pair $(R, S)$ of commuting operators, such that $(R, S) \in \mathcal{O}_{1}^{\prime}$. If $A_{i}, B_{i}(i=1,2)$ are commuting operators on $\mathscr{H}$, then we write the following operators on $H_{F_{1}} \otimes H_{F_{2}}$.

$\phi_{0}(f)=\frac{1}{\sqrt{2}}\left(\varphi_{1}\left(A_{1} f\right)+\varphi_{1}^{*}\left(B_{1} f\right)\right) \otimes 1_{2}+\theta_{1} \otimes \frac{1}{\sqrt{2}}\left(\varphi_{2}\left(A_{2} f\right)+\varphi_{2}^{*}\left(B_{2} f\right)\right)$

$\phi_{0}^{*}(f)=\frac{1}{\sqrt{2}}\left(\varphi_{1}\left(\bar{B}_{1} f\right)+\varphi_{1}^{*}\left(\bar{A}_{1} f\right)\right) \otimes 1_{2}+\theta_{1} \otimes \frac{1}{\sqrt{2}}\left(\varphi_{2}\left(\bar{B}_{2} f\right)+\varphi_{2}^{*}\left(\bar{A}_{2} f\right)\right)$

where $\theta_{1}$ is an operator anticommuting with all creation and annihilation operators $\varphi_{1}^{*}$ and $\varphi_{1}$ on $H_{F_{1}}$. It is easily checked that the operators (30) and (31) generate a representation $\phi$ of $\mathfrak{A}$ on $H_{F_{1}} \otimes H_{F_{2}}$ with cyclic vector $\Omega_{12}=\Omega_{F_{1}} \otimes \Omega_{F_{2}}$ if the operators $A_{i}, B_{i}(i=1,2)$ satisfy

$$
\begin{aligned}
& A_{1}^{*} A_{1}+B_{1}^{*} B_{1}=1 \\
& A_{1}^{\prime} B_{1}+B_{1}^{\prime} A_{1}=0 \\
& A_{2}^{*} A_{2}+B_{2} B_{2}=1 \\
& A_{2}^{\prime} B_{2}+B_{2}^{\prime} A_{2}=0 .
\end{aligned}
$$

The representation $\phi$ corresponds to the quasi-free state $\varrho$ if the operators $A_{i}, B_{i}(i=1,2)$ satisfy furthermore

$$
\begin{aligned}
R & =\frac{1}{2}\left(B_{1}^{*} B_{1}+B_{2}^{*} B_{2}\right) \\
S & =\frac{1}{2}\left(A_{1}^{\prime} B_{1}+A_{2}^{\prime} B_{2}\right) .
\end{aligned}
$$

This can be seen as follows. It is easy to verify that

$$
\begin{gathered}
(f, R g)=\varrho\left(\left[f^{*}\right][g]\right)=\left(\Omega_{12}, \phi\left(\left[f^{*}\right][g]\right) \Omega_{12}\right) \\
(f, S g)=\varrho([f][g])=\left(\Omega_{12}, \phi([f][g]) \Omega_{12}\right)
\end{gathered}
$$

for all $f, g \in \mathscr{H}$. From the properties of Fock representation it follows that the state induced by $\phi$ and $\Omega_{12}$ is quasi-free and hence it is equal to $\varrho$ on all of $\mathfrak{A}$ or

$$
\varrho(A)=\left(\Omega_{12}, \phi(A) \Omega_{12}\right) \text { for all } A \in \mathfrak{A} .
$$

The problem of representing the quasi-free state $\varrho$ in terms of Fock representation is now reduced to proving that for every pair of operators $(R, S) \in \mathcal{O}_{1}$ there exist operators $A_{i}, B_{i}(i=1,2)$ on $\mathscr{H}$, satisfying the equations $(32)-(37)$.

5 Commun. math. Phys., Vol. 7 
We add the following conditions on the operators $A_{i}, B_{i}(i=1,2)$

$$
\begin{aligned}
& \mathscr{D}_{B_{1}^{-1} \bar{A}_{1}} \text { is dense in } \mathscr{H}, \\
& \mathscr{D}_{B_{2}^{-1} \bar{A}_{2}} \text { is dense in } \mathscr{H} .
\end{aligned}
$$

The unitary parts $W_{1}$ and $W_{2}$ of $A_{1}^{\prime} B_{1}$ and $A_{2}^{\prime} B_{2}$ satisfy $W_{1}^{2}=W_{2}^{2}$. (39)

This means that we look for a solution of the equations $(32)-(37)$ within the class of operators satisfying the conditions (38) and (39). We proceed to analyze the problem, beginning with a simple transformation as given in

Lemma 7. Suppose that $(R, S)$ is a pair of commuting operators satisfying (ii'), (iii), and (iv). Then the problem of solving the equations (32)-(39) is equivalent to the decoupled problem consisting in

1) finding couples of pairs $\left(R_{1}, S_{1}=W_{1}\left|S_{1}\right|\right)$ and $\left(R_{2}, S_{2}=W_{2}\left|S_{2}\right|\right)$ such that

- each pair $\left(R_{i}, S_{i}\right),(i=1,2)$ satisfies the conditions of Lemma 4

- the unitary parts $W_{1}$ and $W_{2}$ of $S_{1}$ and $S_{2}$ satisfy $W_{1}^{2}=W_{2}^{2}$

- the following equations hold

and

$$
\begin{aligned}
& R=\frac{1}{2}\left(R_{1}+R_{2}\right) \\
& S=\frac{1}{2}\left(S_{1}+S_{2}\right)
\end{aligned}
$$

2) solving the equations $(32)-(35),(38)$ together with

$$
\begin{aligned}
R_{1} & =B_{1}^{*} B_{1} \\
S_{1} & =A_{1}^{\prime} B_{1} \\
R_{2} & =B_{2}^{*} B_{2} \\
S_{2} & =A_{2}^{\prime} B_{2} .
\end{aligned}
$$

The proof of this Lemma is obvious.

The second problem in Lemma 7 is equivalent to solving the equations (32), (33), (38a), (42) (43) and (34), (35), (38b) (44), (45). The solution of these two systems of equations is given by Lemma 6 . Our problem is therefore reduced to finding the solution of the first problem of Lemma 7.

Making use of Lemma 4 and the polar decomposition $S=W|S|$ of $S$ we can write (41) as

$$
2|S|=W^{*} W_{1} \sqrt{R_{1}\left(1-R_{1}\right)}+W^{*} W_{2} \sqrt{R_{2}\left(1-R_{2}\right)} \geqq 0 .
$$

First of all we suppose

$$
W_{1}^{2}=W_{2}^{2}=W^{2} .
$$

It may be checked that this assumption does not restrict the class of states we want to represent. It means that $W=W_{1}=W_{2}$ on a certain subspace of $\mathscr{H}$ and that $W= \pm W_{1}=\mp W_{2}$ on the orthogonal complement. 
It remains to solve the following equations for $R_{1}$ and $R_{2}$

$$
\begin{gathered}
R_{1}^{*}=R_{1} ; R_{2}^{*}=R_{2} \\
0 \leqq R_{1} \leqq 1 ; 0 \leqq R_{2} \leqq 1 \\
2 R=R_{1}+R_{2} \\
2|S|=W^{*} W_{1} \sqrt{R_{1}\left(1-R_{1}\right)+W^{*} W_{2} \sqrt{R_{2}\left(1-R_{2}\right)}}
\end{gathered}
$$

where $R_{1}$ and $R_{2}$ commute and the unitary operators $W, W_{1}$ and $W_{2}$ satisfy (46).

We make the transformation

$$
R_{1}=\frac{1+E_{1}}{2} ; R_{2}=\frac{1+E_{2}}{2}
$$

and obtain the following system of equations for $E_{1}$ and $E_{2}$ equivalent to $(46)-(49)$.

$$
\begin{gathered}
E_{1}^{*}=E_{1} ; E_{2}^{*}=E_{2} \\
-1 \leqq E_{1} \leqq 1 ;-1 \leqq E_{2} \leqq 1 \\
4 R-2=E_{1}+E_{2} \\
4|S|=W^{*} W_{1} \sqrt{1-E_{1}^{2}}+W^{*} W_{2} \sqrt{1-E_{2}^{2}}
\end{gathered}
$$

Set

$$
\begin{gathered}
F_{1}=W^{*} W_{1} \sqrt{1-E_{1}^{2}}+i E_{1} ; F_{2}=W^{*} W_{2} \sqrt{1-E_{2}^{2}}+i E_{2} \\
K=4|S|+i(4 R-2) .
\end{gathered}
$$

Then (53) and (54) can be written in the form

$$
K=F_{1}+F_{2} \text {. }
$$

Notice that from (46) it follows that $F_{1}$ and $F_{2}$ are unitary operators. Let $K=U P$ be the polar decomposition of the operator $K(56)$. Since $R$ and $S$ commute, the operator $K$ is normal, hence $U$ may be taken unitary and such that $U$ commutes with $P$ and all operators commuting with $K$ and $K^{*}$. The equation (57) is equivalent to

$$
U * F_{1}+U * F_{2}=P
$$

where $U^{*} F_{1}$ and $U^{*} F_{2}$ are unitary operators.

Notice furthermore that

$$
P^{2}=(4 R-2)^{2}+(4|S|)^{2}
$$

and that $P=0$ is equivalent to $R=\frac{1}{2}, S=0$. In this case the decomposition (40) and (41) is found to be

$$
\begin{aligned}
& R=\frac{1}{2} \cdot 0+\frac{1}{2} \cdot 1 \\
& S=0+0 .
\end{aligned}
$$

In the general case $\mathscr{H}=\mathscr{H}_{1} \oplus \mathscr{H}_{1}^{\perp}$, where $\mathscr{H}_{1}=\eta_{P}$. We have $\eta_{P}=\eta_{|S|} \cap \eta_{R-\frac{1}{2}}$. Because $[R, S]=0, \mathscr{H}_{1}$ is invariant under 
$|S|, R$ and $P$ and so its orthogonal complement $\mathscr{H}_{1}^{\frac{1}{1}}$. The positive operator $P$ has an inverse $P^{-1}$ on $\mathscr{H}_{1}^{\perp}$, defined on the dense subset $\mathscr{R}_{P}$ where $P^{-1}$ is not necessarily bounded. On $\mathscr{H}_{1}$ the solution of (40) and (41) is given by (60) and (61). On $\mathscr{H}_{1}^{\frac{1}{1}}$ the solution of (58) is

$$
\begin{aligned}
& U^{*} F_{1}=\frac{P}{2}+i \alpha \sqrt{1-\frac{P^{2}}{4}} \\
& U^{*} F_{2}=\frac{P}{2}-i \alpha \sqrt{1-\frac{P^{2}}{4}}
\end{aligned}
$$

where $\alpha$ is a constant such that $\alpha= \pm 1$; both values of $\alpha$ give the same solution, therefore from now on we put $\alpha=1$. Using the expression (59) it follows immediately from the positivity condition that

$$
1-\frac{P^{2}}{4} \geqq 0 \text {. }
$$

From (50), (54), (55), (62) and (63) we obtain the final result for the decomposition (40) and (41).

$$
\begin{aligned}
& S_{1}=S-W \sqrt{\frac{4-P^{2}}{4 P^{2}}}(2 R-1) \\
& S_{2}=S+W \sqrt{\frac{4-P^{2}}{4 P^{2}}}(2 R-1) \\
& R_{1}=R+|S| \sqrt{\frac{4-P^{2}}{4 P^{2}}} \\
& R_{2}=R-|S| \sqrt{\frac{4-P^{2}}{4 P^{2}}} .
\end{aligned}
$$

If the operator $P^{-1}$ is not bounded on $\mathscr{H}_{1}^{\frac{1}{1}}$, it may however be shown that the operators defined in $(64)-(67)$ are bounded, therefore they are defined on the wohle space by continuity. It remains to verify that (64) - (67) actually satisfy (47) - (49).

The conditions (47a), (48) and (49) are clearly satisfied; let us finally verify the condition $(47 \mathrm{~b})$; we set

$$
H=|S| \sqrt{\frac{4-P^{2}}{4 P^{2}}} .
$$

Because $R$ and $S$ commute $H \geqq 0$ and it remains to prove

which is equivalent to

$$
R+H \leqq 1 ; 0 \leqq R-H
$$

$$
(1-R)^{2}-H^{2} \geqq 0 ; R^{2}-H^{2} \geqq 0 .
$$

An elementary calculation shows

$$
\begin{aligned}
(1-R)^{2}-H^{2} & =\frac{4}{P^{2}}\left[2|S|^{2}-2 R(1-R)+1-R\right]^{2} \\
R^{2}-H^{2} & =\frac{4}{P^{2}}\left[2|S|^{2}-2(1-R) R+R\right]^{2} .
\end{aligned}
$$

Therefore (69) and consequently $(47 \mathrm{~b})$ is true. 
As indicated above we use now the solution of the equations of Lemma 6 to solve completely the problem of representing the state $\varrho$ on $H_{F_{1}} \otimes H_{F_{2}}$. We formulate the result in

Theorem 4. Suppose that $(R, S)$ is a commuting pair of operators satisfying the conditions of theorem 2 then the equations $(32)-(39)$ have the following solution

$$
\begin{aligned}
& A_{1}=-V^{* \prime} W_{1} \sqrt{1-R-H} \\
& B_{1}=V \sqrt{R+H} \\
& A_{2}=-V^{*} W_{2} \sqrt{1-R+H} \\
& B_{2}=V \sqrt{R-H}
\end{aligned}
$$

where $H$ is given in (68), and $V$ is an arbitrary unitary operator.

The quasi-free state $\varrho$, defined by $R$ and $S$ corresponds to the representation $\phi$ on $H_{F_{1}} \otimes H_{F_{2}}$ defined by $(30)$ and (31) with the expressions (70)-(73) substituted for $A_{1}, B_{1}, A_{2}$ and $B_{2}$. The representation $\phi i s$ a cyclic and reducible factor representation.

Proof. Everything has been proved, except the last statement. We omit the standard proof of the fact, that $\phi$ is cyclic [3]. Let us prove that $\phi$ is reducible. Let $\phi^{\prime}$ be the representation of $\mathfrak{A}$ on $H_{F_{1}} \otimes H_{F_{2}}$ defined by

$$
\begin{aligned}
\phi_{0}^{\prime}(f) & =\frac{\theta_{1}}{\sqrt{2}}\left(\varphi_{1}\left(A_{1} f\right)+\varphi_{1}^{*}\left(B_{1} f\right)\right) \otimes \theta_{2}+\mathbf{1}_{1} \otimes \frac{1}{\sqrt{2}}\left(\varphi_{2}\left(A_{2} f\right)+\varphi^{*}\left(B_{2} f\right)\right) \theta_{2} \\
\phi_{0}^{\prime *}(f) & =\frac{1}{\sqrt{2}}\left(\varphi_{1}\left(\bar{B}_{1} f\right)+\varphi_{1}^{*}\left(\bar{A}_{1} f\right)\right) \theta_{1} \otimes \theta_{2}+\mathbf{1}_{1} \otimes \frac{\theta 2}{\sqrt{2}}\left(\varphi_{2}\left(\bar{B}_{2} f\right)+\varphi_{2}^{*}\left(\bar{A}_{2} f\right)\right) .
\end{aligned}
$$

One verifies

$$
\left[\phi_{0}(f), \phi_{0}^{\prime}(g)\right]=\left[\phi_{0}^{*}(f), \phi_{0}^{\prime}(g)\right]=\left[\phi_{0}(f), \phi_{0}^{\prime *}(g)\right]=\left[\phi_{0}^{*}(f), \phi_{0}^{\prime *}(g)\right]=0 \text {. }
$$

Hence $\phi^{\prime}(\mathfrak{U}) \subseteq[\phi(\mathfrak{U})]^{\prime}$ therefore the commutant $[\phi(\mathfrak{U})]^{\prime}$ is not trivial, and the representation $\phi$ is reducible.

Next we prove that $\phi$ is a factor representation, i.e. that the von Neumann algebra $L$ generated by $\phi(\mathfrak{A})$ is a factor.

A simple calculation shows that ${ }^{3}$

where

$$
\begin{aligned}
& \phi_{0}(f)-\phi_{0}^{\prime}(f) \theta_{1} \otimes \theta_{2}=\chi_{1}(f) \otimes \mathbf{1}_{2} \\
& \phi_{0}(f)+\phi_{0}^{\prime}(f) \theta_{1} \otimes \theta_{2}=\theta_{1} \otimes \chi_{2}(f)
\end{aligned}
$$

$$
\begin{aligned}
& \chi_{1}(f)=\sqrt{2}\left(\varphi_{1}\left(A_{1} f\right)+\varphi_{1}^{*}\left(B_{1} f\right)\right) \\
& \chi_{2}(f)=\sqrt{2}\left(\varphi_{2}\left(A_{2} f\right)+\varphi_{2}^{*}\left(B_{2} f\right)\right) .
\end{aligned}
$$

Let $Q$ be an operator commuting with $\left\{L \cup L^{\prime}\right\}^{\prime \prime}$ and let $L_{1}$ and $L_{2}$ be the von Neumann algebras generated by $\left\{\chi_{1}(f) \otimes \mathbf{1}_{2}, \chi_{1}^{*}(f) \otimes \mathbf{1}_{2}\right\}$ and $\left\{\theta_{1} \otimes \chi_{2}(f), \theta_{1} \otimes \chi_{2}^{*}(f)\right\}$ respectively. The subalgebra $L_{1}$ is irreducible in

\footnotetext{
${ }^{3}$ It should be verified that $\theta_{1} \otimes \theta_{2} \in L \cup L^{\prime}$.
} 
the first space $H_{F_{1}} \sim H_{F_{1}} \otimes \Omega_{F_{2}}$ and therefore $Q$ is of the form $\lambda \mathbf{1}_{1} \otimes Q_{1}$. The subalgebra $L_{2}$ is irreducible in the second space $H_{F_{2}} \sim \Omega_{F_{1}} \otimes H_{F_{2}}$ and therefore $Q$ is of the form $\lambda \mathbf{1}_{1} \otimes \mathbf{1}_{2}$. Consequently each operator $Q \in\left(L \cup L^{\prime}\right)^{\prime}=L \cap L^{\prime}$ is of the form $\lambda \mathbf{1}$ or $L \cap L^{\prime}=\{\mathbb{C} \mathbf{1}\}$ and $L$ is a factor.

\section{The Case $\boldsymbol{R}, \mathbf{1}-\boldsymbol{R}$ and $\boldsymbol{S}$ Invertible}

In this section we treat another class of quasi-free states. We do not assume commutativity, but introduce another simplifying assumption, that $R, 1-R$ and $S$ are invertible. Let us introduce for simplicity the following notation

Definition $8 . \mathcal{O}_{\mathrm{I}}=\left\{(R, S) \in \mathcal{O} \mid R^{-1},(1-R)^{-1}\right.$ and $S^{-1}$ are bounded operators on $\mathscr{H}\}$.

First we characterize the set $\mathcal{O}_{\mathrm{I}}$ in the following Lemma.

Lemma 8. $(R, S) \in \mathcal{O}_{\mathrm{I}}$ if and only if

(ii) $X=1-R^{\prime}-S R^{-1} S^{*} \geqq 0$

(iii) $R=R^{*} ; 0 \leqq \varepsilon \leqq R \leqq 1-\varepsilon, \varepsilon>0$

(iv) $S^{\prime}=-S ; S$ is invertible.

Proof. We notice that $\mathscr{D}_{R^{-1} S^{*}}=\mathscr{D}_{\left(1-R^{\prime}\right)^{-1} S}=\mathscr{H}$ and the Lemma follows from Theorem 2 . It can also be verified directly, that $X \geqq 0$ if and only if $Y \geqq 0$.

$$
\text { A. The case } X=0
$$

The set of pairs of operators $(R, S) \in \mathcal{O}_{1}$ with $X=0$ can be described as follows.

Lemma 9. $(R, S) \in \mathcal{O}_{\mathrm{I}}$ and $X=0$ if and only if

$$
\begin{gathered}
R=R^{*} ; 0<\varepsilon \leqq R \leqq 1-\varepsilon \\
W W^{*}=W^{*} W ; W^{\prime}=-W . \\
W R=R^{\prime} W \\
|S|=\sqrt{R(1-R) ; S=W|S| .}
\end{gathered}
$$

Proof. It is easy to verify, that if $(R, S)$ satisfy $(74)-(77)$, then $S^{\prime}=-S ; S, R$ and $1-R$ are invertible and $X=0$. Suppose on the other hand that $R$ and $S$ satisfy (iii), (iv) and $X=0$ and $(R, S) \in \mathcal{O}_{\mathrm{I}}$. Introducing the polar decomposition $S=W|S|$ of $S$ in the equations $X=0$ and $X^{\prime}=0$, and setting $Z=W^{*} R^{\prime} W$, we arrive at the equations

$$
\begin{aligned}
& 1-Z=|S| R^{-1}|S| \\
& 1-R=|S| Z^{-1}|S| .
\end{aligned}
$$

The solution of these equations is

hence

$$
Z=R ;|S|=\sqrt{R(1-R)}
$$

$$
W R=R^{\prime} W .
$$


From (iv) and this result follows finally

$$
W^{\prime}=-W \text {. }
$$

Lemma 5 holds for $(R, S) \in \mathcal{O}_{\mathrm{I}}$ with the only change, that the assumption that $\mathscr{D}_{B^{-1} \bar{A}}$ is dense in $\mathscr{H}$ is replaced by the condition that $A$ and $B$ be invertible.

Concerning the solution of equations $(23)-(26)$ given in Lemma 6 we notice that the solution given by (28), (29) is complete for $(R, S) \in \mathcal{O}_{\mathrm{I}}$. This can easily be verified by using the polar decompositions of $A$ and $B$.

Consequently theorem 3 is valid for $(\mathrm{R}, S) \in \mathcal{O}_{\mathrm{I}}$ and $X=0$, and all the representations of the form $(21),(22)$ of a given state $\varrho \in Q-\mathscr{F}_{\text {I }}$ are parametrized by the unitary operator $V$ of Lemma 6 .

Example. Let $\mathscr{H}=\mathscr{H}_{1} \oplus \mathscr{H}_{2}$, and let $\mathscr{I}$ be a unitary operator from $\mathscr{H}_{1}$ to $\mathscr{H}_{2}$. Let $R_{0}$ be a self-adjoint operator on $\mathscr{H}_{1}, 0<\varepsilon \leqq R_{0} \leqq 1-\varepsilon$, and set

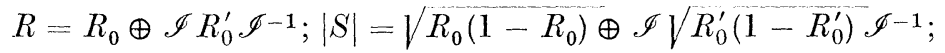

$$
\begin{aligned}
& W(f \oplus g)=\mathscr{I}^{-1} g \oplus \mathscr{I} f \text {. }
\end{aligned}
$$

Then the pair of invertible operators $(R, S=W|S|)$ satisfy $X=0$, (iii) and (iv). The solution of the equations (20)-(23) is given by

$$
\begin{aligned}
A(f \oplus g) & =\mathscr{I}^{-1} \sqrt{1-\mathscr{I} R_{0}^{\prime} \mathscr{I}^{-1}} g \oplus\left(-\mathscr{I} \sqrt{1-R_{0} f}\right) \\
B & =\sqrt{R_{0}} \oplus \sqrt{\mathscr{I} R_{0}^{\prime} \mathscr{I}^{-1}} .
\end{aligned}
$$

\section{B. The case $X \geqq 0, X \neq 0$}

First of all, we impose supplementary conditions on the states $\varrho$ which are automatically satisfied when $[R, S]=0$ and $\left[S, S^{*}\right]=0$. We formulate these as the following conditions on $R$ and $S=W|S|$

(v) $W^{\prime}=-W$

(vi) $W R=R^{\prime} W$

(vii) $W|S|=|S|^{\prime} W$

(viii) $0<\varepsilon \leqq R+H \leqq 1-\varepsilon$

$$
0<\varepsilon \leqq R-H \leqq 1-\varepsilon
$$

where

$$
H=\frac{1}{4}\left[U \sqrt{-\frac{P^{2}}{4}}+\sqrt{1-\frac{P^{2}}{4}} U^{*}\right]
$$

and $U$ and $P$ are the unitary and positive part of the invertible operator

$$
K=4|S|+i(4 R-2) .
$$

We notice that in the commutative case (v) - (vii) are proved in (15), (16), (18); the expression for $H$ takes the simpler form given in (68) and (viii) is proved in (69). It is likely, that (viii) can be proved also in the present case, but (v) - (vii) have to be imposed as conditions. 
The representation of section IV. B given in (30), (31) is also valid under the present conditions: $(R, S) \in \mathcal{O}_{\mathrm{I}}$ and the condition $(38)$ is replaced by the requirement that $A_{i}, B_{i}(i=1,2)$ be invertible. Hence in order to represent $\varrho$ on $H_{F_{1}} \otimes H_{F_{2}}$ it suffices to solve the equations (32)-(37), (39) under these conditions. We transform this problem as in Lemma 7, but with the present conditions, and the solution of the equations is obtained by the same method. The possibility of applying this method is based on the polar decomposition of the operators $S$ and $K$, and here it is essential, that $W$ and $U$ are unitary operators. In the present case this follows from the assumption of invertibility. Also, the assumptions (v)-(viii) are essential for the solution of (40) and (41). The explicit solutions of these equations are in the non-commutative case given by the expressions

$$
\begin{aligned}
& S_{1}=S+L \\
& S_{2}=S-L \\
& R_{1}=S+H \\
& R_{2}=S-H
\end{aligned}
$$

where $H$ is given by (78), (79) and

$$
L=W \frac{i}{4}\left[U \sqrt{1-\frac{P^{2}}{4}}-\sqrt{1-\frac{P^{2}}{4}} U^{*}\right] .
$$

As a consequence of the predecing discussion, we conclude that theorem 4 is valid under the assumptions (ii) - (viii) on the pair $(R, S)$ with $H$ defined in (78), (79).

Remark. It is possible to obtain a representation on $H_{F_{1}} \otimes H_{F_{2}}$ of quasi-free states $\varrho$, which do not satisfy the condition of commutativity or invertibility, by the method developed above.

Such states can, for example, be constructed as follows.

Suppose that $\left(R_{i}, S_{i}\right) \in \mathcal{O}_{1}^{\prime}, X_{i}=0, \mathscr{R}_{R_{i}}$ closed, $W_{i}$ unitary $(i=1,2)$ and set

$$
R=\frac{1}{2}\left(R_{1}+R_{2}\right) ; S=\frac{1}{2}\left(S_{1}+S_{2}\right)
$$

then $(R, S) \in \mathcal{O}_{1}^{\prime}$, and $X=0$ if and only if $R_{1}=R_{2} ; S_{1}=S_{1}$.

Proof. It is clear that $(R, S)$ satify (iii) and (iv). Moreover $\mathscr{D}_{R^{-1} S^{*}}=\mathscr{H}$, and $(R, S) \in \mathfrak{O}_{1}^{\prime}$. If $X=0$, then the form

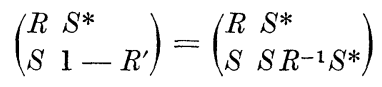

is equal to zero on the pairs of vectors $\left\{\left(-R^{-1} S^{*} g, g\right) \mid g \in \mathscr{H}\right\}$. Because

$$
\left(\begin{array}{ll}
R & S^{*} \\
S & 1-R^{\prime}
\end{array}\right)=\frac{1}{2}\left(\begin{array}{ll}
R_{1} & S^{*} \\
S_{1} & S_{1} R_{1}^{-1} S_{1}^{*}
\end{array}\right)+\frac{1}{2}\left(\begin{array}{ll}
R_{2} & S_{2}^{*} \\
S_{2} & S_{2} R_{2}^{-1} S_{2}^{*}
\end{array}\right)
$$

the two forms have to vanish on the same elements. It follows that $R_{1}^{-1} S_{1}^{*}=R_{2}^{-1} S_{2}^{*}$ hence $W_{1}=W_{2}, R_{1}=R_{2}$ and $S_{1}=S_{2}$. Conversely, if $R_{1}=R_{2}, S_{1}=S_{2}$ then immediately $X=0$. 


\section{An Application of Invariant Quasi-free States}

In this section we give an application of invariant quasi-free states of fermion systems with spin. Actually in the foregoing section we did not introduce the spin. However all the results mentioned before can be obtained if we consider spin $\frac{1}{2}$ nonrelativistic particles.

First we define the $\mathrm{C}^{*}$-Clifford algebra in this case

Definition 9. $\mathfrak{A}_{s}(\mathfrak{M})$, the Clifford algebra over $\mathfrak{M}$, is the algebra generated by the monomials $\left[h_{1}\right]_{s_{1}} \ldots\left[h_{n}\right]_{s_{n}} ; h_{i} \in \mathfrak{M} \cup \mathfrak{M}^{*}, 1 \leqq i \leqq n$; $\left(s_{i}=1\right.$ or $2 ; s_{i}$ is the spin index), and the unit element 1 , such that

$$
\begin{gathered}
\left\{[f]_{i},[g]_{i}\right\}_{+}=0 ;\left\{[f]_{i},\left[g^{*}\right]_{i}\right\}=(g, f) \mathbf{1} \quad i=1,2 \\
c_{1}[f]_{i}+c_{2}[g]_{i}-\left[c_{2} f+c_{2} g\right]_{i}=0 \quad f, g \in \mathfrak{M} . \\
\left\{[h]_{1},[g]_{2}\right\}_{+}=0 \quad h, g \in \mathfrak{M} \cup \mathfrak{M}^{*} .
\end{gathered}
$$

The involution on $\mathfrak{A}_{s}(\mathfrak{M})$ is defined as in definition 1 .

Fock representation of $\mathfrak{A}_{s}(\mathfrak{W})$ again induces a norm $\|$. $\|$ on the algebra, such that the closure $\mathfrak{A}_{s}$ of $\mathfrak{A}_{s}(\mathfrak{M})$ with respect to this norm is a $\mathrm{C}^{*}$ algebra.

Now we proceed to analyse a particular class of quasi-free invariant states on $\mathfrak{A}_{s}$. For reasons of technical convenience we introduce the field variables as distributions $\varphi_{r}(x)$ and $\varphi_{r}^{*}(x), r=1,2$ with values in $\mathfrak{A}_{s}$ defined by

where $x \in R^{3}$.

$$
\begin{aligned}
\varphi_{r}(f) & =\int f(x) \varphi_{r}(x) d x \\
\varphi_{r}^{*}(f) & =\int \overline{f(x)} \varphi_{r}^{*}(x) d x
\end{aligned}
$$

Moreover we use the following notation

Definition 10. Let $W$ be the set of invariant, quasi-free states $\varrho$ on $\mathfrak{A}_{s}$, such that

$$
\begin{array}{ll}
\varrho\left(\varphi_{r}^{*}(x) \varphi_{s}(y)\right)=0 & \text { for } \quad r \neq s \\
\varrho\left(\varphi_{i}(x) \varphi_{i}(y)\right)=0 & \text { for } \quad i=1,2 .
\end{array}
$$

It is clear that $W$ is a compact subset of $\mathfrak{A}_{s^{3}}^{\prime+}$ in the weak *-topology.

The states $\varrho \in W$ are determined by pairs of convolution operators $R$ and $S$ corresponding to distributions $r$ and $s$ defined by

$$
\begin{aligned}
& r(x-y)=\varrho\left(\varphi_{1}^{*}(x) \varphi_{1}(y)\right)=\varrho\left(\varphi_{2}^{*}(x) \varphi_{2}(y)\right) \\
& s(x-y)=\varrho\left(\varphi_{1}(x) \varphi_{2}(y)\right)=\varrho\left(\varphi_{2}(x) \varphi_{1}(y)\right) .
\end{aligned}
$$

For simplicity we work in momentum space with the Fourier transforms $\tilde{r}(p)$ and $\tilde{s}(p)$ of $r$ and $s$. 
Suppose that the interaction between the Fermion particles is given by the central symmetric two-body interaction potential $V(x)$, where $V(x) \in L^{1}\left(R^{3}\right)$. We assume as Hamiltonian density in the finite volume:

$$
\begin{gathered}
V_{L}=\left\{x \in R^{3} \mid-\frac{L}{2} \leqq x_{i} \leqq \frac{L}{2} ; i=1,2,3,\right\} \\
\mathscr{H}_{L}(x)=\sum_{i=1}^{2} \nabla \varphi_{i}^{*}(x) \nabla \varphi_{i}(x)+\int_{V_{L}} \varphi_{1}^{*}(x) \varphi_{2}^{*}(y) V(x-y) \varphi_{1}(x) \varphi_{2}(y) d y .
\end{gathered}
$$

The number density operator is

We define

$$
\mathscr{N}(x)=\sum_{i=1}^{2} \varphi_{i}^{*}(x) \varphi_{i}(x) .
$$

$$
H_{c}\left(V_{L}\right) \frac{1}{L^{3}} \int_{V_{L}}\left\{\mathscr{H}_{L}(x)-\mu \mathscr{N}(x)-c\right\} d x
$$

where $\mu$ is the chemical potential and $c$ a real number. We assume, that $V(x)$ satisfies furthermore the condition that there exists a constant $c$ such that

$$
\varrho\left(H_{c}\left(V_{L}\right)\right) \geqq 0 \quad \text { for every } \quad 0<L<\infty, \varrho \in W .
$$

This condition guarantees that for the finite system the spectrum of the Hamiltonian is bounded from below.

Set

$$
\varrho(H)=\lim _{L \rightarrow \infty} \varrho\left(\frac{1}{L^{3}} \int_{V_{L}}\{\mathscr{H}(x)-\mu \mathscr{N}(x)\} d x\right) .
$$

It follows from the assumption that $V(x) \in L^{1}\left(R^{3}\right)$, that $\varrho(H)$ is a well defined quantity for any $\varrho \in W$.

Definition 11. The ground state energy $c_{0}$ of the infinite system is

$$
c_{0}=\inf _{\varrho \in W} \varrho(H)
$$

and let $M=\left\{\varrho \in W \varrho(H)=c_{0}\right\}$ be the set of ground states.

Since $\varrho(H)$ is a continuous function on the compact set $W$, it follows that $M$ is a non-empty compact set.

The particle density $d_{\varrho}$ in the state $\varrho \in W$ is defined as

$$
d_{\varrho}=\lim _{L \rightarrow \infty} \frac{1}{L^{3}} \int_{V_{L}} \tilde{r}(p) d p
$$

when the limit exists. We denote by $M_{0}$ the set of states $\varrho \in M$ such that $d_{\varrho}$ is defined. It is clear, that also $M_{0}$ is a non-empty compact set. In what follows we determine $M_{0}$ by a variational procedure.

In momentum space (82) becomes

$$
\begin{aligned}
\varrho(H)= & 2 \int_{R^{3}}\left(p^{2}-\mu\right) \tilde{r}(p) d p- \\
& -(2 \pi)^{3} \int_{R^{3} \times R^{3}}\left\{\widetilde{V}(0) \tilde{r}(q) \tilde{r}(p)+\widetilde{V}(p-q) \tilde{s}^{*}(p) \tilde{s}(q)\right\} d q d p .
\end{aligned}
$$


The problem of minimizing $\varrho(H)$ over $W$ is equivalent to the problem of minimizing $\varrho(H)$ as given by (83) over the set of pairs $(\tilde{r}, \tilde{s})$, such that $(\tilde{r}, \tilde{s}) \in L^{\infty}\left(R^{3}\right)$ and

$$
\begin{gathered}
0 \leqq \tilde{r}(p) \leqq 1 \\
\tilde{s}(-p)=-\tilde{s}(p) \\
\tilde{\tau}^{2}(p)=\tilde{r}(p)(1-\tilde{r}(-p))-|\tilde{s}(p)|^{2} \geqq 0 .
\end{gathered}
$$

It is convenient to perform the variation with respect to $\sqrt{\tilde{r}}(p), \tau(p)$ and $\alpha(p)$, where $\alpha(p)=\frac{1}{2} \arg \tilde{s}(p)$.

This leads to the following system of equations

$$
\begin{aligned}
& \sqrt{\tilde{r}(p)}\left\{2\left(p^{2}-\mu\right)-(2 \pi)^{3}\right. \\
& \left.\int\left\{2 \widetilde{V}(0) \tilde{r}(q)+\widetilde{V}(p-q) \frac{1-2 \tilde{r}(p)}{|\tilde{s}(p)|^{2}} \operatorname{Re} \tilde{s}^{*}(p) \tilde{s}(q)\right\} d q\right\}=0 \\
& \quad \tilde{\tilde{\tau}(p)} \int \widetilde{V}(p-q) \operatorname{Re} \tilde{s}^{*}(p) \tilde{s}(q) d q=0 \\
& \quad \int \widetilde{s}\left(\left.p\right|^{2}(p-q) \operatorname{Im} \tilde{s}^{*}(q) \tilde{s}(p) d q=0 .\right.
\end{aligned}
$$

The analysis of this system of equations yields the following characterization of the set $M_{0}$ of ground states with well defined particle density.

The pair of functions $\tilde{r}(p) ; \tilde{s}(p)$ satisfying $(84)-(86)$ define a state $\varrho \in M_{0}$ if and only if

$$
\begin{gathered}
\tau(p)^{2}=\tilde{r}(p)(1-\tilde{r}(p))-|\tilde{s}(p)|^{2}=0 \\
\alpha(p)=\frac{1}{2} \arg \tilde{s}(p)=\alpha=\mathrm{constan} t
\end{gathered}
$$

and the function

$$
\varphi_{\alpha}(p)=(2 \pi)^{3} \int \widetilde{V}(p-q) \tilde{s}(q) e^{i \alpha} d q
$$

satisfies the integral equation

where

$$
\varphi_{\alpha}(p)=\frac{1}{2} \int \frac{\widetilde{V}\left(p-p^{\prime}\right) \varphi_{\alpha}\left(p^{\prime}\right) d p^{\prime}}{\left[\left(p^{\prime 2}-\mu^{\prime}\right)^{2}+\left|\varphi_{\alpha}\left(p^{\prime}\right)\right|^{2}\right]^{1 / 2}}
$$

$$
\mu^{\prime}=\mu+(2 \pi)^{3} d_{\varrho} \widetilde{V}(0)
$$

Equation (89) is an integral equation of the type found for the energy gap in the Bardeen-Cooper-Schriefer model for superconductivity and the constant $\mu^{\prime}$ is the chemical potential corrected by its first order Born approximation.

If $\varphi_{0}(p)$ is a solution of (89), one verifies that also $\varphi_{\alpha}(p)=e^{i \alpha} \varphi_{0}(p)$ is a solution and that the energy density $c_{0}$ is the same for the whole one-parameter manifold of solutions $\left\{\varphi_{\alpha}(p)=e^{i \alpha} \varphi_{0}(p) \mid 0 \leqq \alpha<2 \pi\right\}$.

It follows from equation (87) that the ground states of the infinite system, mediated by the two-body potential $V(x)$, correspond to irreducible representations. 
Finally let us remark that we do not claim to have obtained a very good approximation for the ground state energy for the model treated here, whereas for the B.C.S. model one may hope to find the exact solution within the class of quasi-free states.

Acknowledgements. We are indebted to D. R. RoBisson for many fruitful discussions during the initial stage of the present work, to N. HuGENHOLTz for interesting comments on the application of the theory, and to D. KASTLER for reading the manuscript and suggesting important improvements in the final version.

We also want to express our gratitude to L. Motchane for his kind hospitality at the I. H. E. S.

\section{References}

1. Kastler, D.: Commun. Math. Phys. 1, 14 (1965).

2. Robinson, D. D.: Commun. Math. Phys. 1, 159-174 (1965).

3. Araki, H., and W. Wyss: Helv. Phys. Acta 37, 136 (1964).

4. Shale, D., and W. F. Stinespring: Ann. Math. 80, 365 (1964).

5. HAAG, R.: Nuovo Cimento 25, 287 (1962).

6. Guichardet, M.: Ann. Ecol. Norm. Super. 3e série t. 83, 1 (1966). Cordesse, A., et G. Rideau: Nuovo Cimento 45, 1 (1966).

7. LANFORD, O., and D. RUELLE: Integral representations of invariant states on $B^{*}$-algebras. Preprint.

8. Strreater, R., and A. Wightman: P. C. T., spin and statistics, and all that. New York: Benjamin 1964.

9. Doplicher, S., R. V. Kadison, D. Kastler, and D. W. Robinson: Asymptotically Abelian systems. Preprint.

10. Hörmander, L.: Acta Math. 104, 93 (1960).

11. DUnford, N., and J. T. Schwartz: Linear operators, part II, p. 935. New York: Interscience 1963. 